Check for updates

Cite this: Phys. Chem. Chem. Phys., 2018, 20, 29142

Received 17th August 2018 Accepted 31st October 2018

DOI: $10.1039 / c 8 c p 05254 a$

rsc.li/pccp

\title{
Reaction of porphyrin-based surface-anchored metal-organic frameworks caused by prolonged illumination $\dagger$
}

\author{
Michael Adams, (D) ${ }^{a}$ Nicolò Baroni, ${ }^{a}$ Michael Oldenburg, ${ }^{a}$ Felix Kraffert, (D) ${ }^{b}$ \\ Jan Behrends, (D) ${ }^{b}$ Rowan W. MacQueen, (D) ${ }^{c}$ Ritesh Haldar, (D) d Dmitry Busko, ${ }^{a}$ \\ Andrey Turshatov, (D) ${ }^{a}$ Ganapathi Emandi, ${ }^{e}$ Mathias O. Senge, (D) e Christof Wöll, (D) d \\ Klaus Lips, (D) bf Bryce S. Richards (D) ag and lan A. Howard (D) *ag
}

\begin{abstract}
Crystalline surface-anchored metal-organic framework (SURMOF) thin films made from porphyrin-based organic linkers have recently been used in both photon upconversion and photovoltaic applications. While these studies showed promising results, the question of photostability in this organic-inorganic hybrid material has to be investigated before applications can be considered. Here, we combine steadystate photoluminescence, transient absorption, and time-resolved electron paramagnetic resonance spectroscopy to examine the effects of prolonged illumination on a palladium-porphyrin based SURMOF thin film. We find that phototreatment leads to a change in the material's photoresponse caused by the creation of stable products of photodecomposition - likely chlorin - inside the SURMOF structure. When the mobile triplet excitons encounter such a defect site, a short-lived ( $80 \mathrm{~ns}$ ) cation-anion radical pair can be formed by electron transfer, wherein the charges are localized at a porphyrin and the photoproduct site, respectively.
\end{abstract}

\section{Introduction}

The photophysics of ordered porphyrin arrays has been a topic of longstanding interest, prompted by their use as analogues of natural light-harvesting systems ${ }^{1-3}$ as well as in photoconductive and photovoltaic device structures. ${ }^{4}$ Also, exciting early work established the ability of the ordered columnar structures of porphyrin chromophores - formed either in a liquid-crystal or solid phase - to efficiently conduct charges. This transport is brought about by electron hopping between molecules and was first observed by time-resolved microwave conductivity (TRMC)

\footnotetext{
${ }^{a}$ Institute of Microstructure Technology, Karlsruhe Institute of Technology, Eggenstein-Leopoldshafen, Germany.E-mail: ian.howard@kit.edu

${ }^{b}$ Fachbereich Physik, Freie Universität Berlin, Berlin Joint EPR Lab, Berlin, Germany

${ }^{c}$ Institute for Nanospectroscopy, Helmholtz-Zentrum Berlin für Materialien und Energie GmbH, Berlin, Germany

${ }^{d}$ Institute of Functional Interfaces, Karlsruhe Institute of Technology, Eggenstein-Leopoldshafen, Germany

${ }^{e}$ School of Chemistry, Trinity Biomedical Sciences Institute, Trinity College Dublin, The University of Dublin, Dublin, Ireland

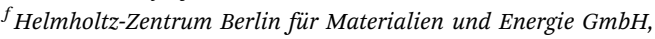
Berlin Joint EPR Lab, Berlin, Germany

${ }^{g}$ Light Technology Institute, Karlsruhe Institute of Technology, Karlsruhe, Germany $\dagger$ Electronic supplementary information (ESI) available. See DOI: 10.1039/ c8cp05254a
}

after excitation with ionizing radiation (3 MeV electrons). ${ }^{5}$ Since then, a wide variety of optoelectronic devices have been demonstrated using porphyrins, which range from memory devices based on photoinduced charge trapping, ${ }^{6}$ to nanowire photoconductors ${ }^{7,8}$ and dye-sensitized solar cells. ${ }^{9,10}$ With regard to the latter, significant study has gone into understanding the nature of charge generation in films of porphyrins deposited on titanium dioxide $\left(\mathrm{TiO}_{2}\right){ }^{11,12}$ In the case of sensitized $\mathrm{TiO}_{2}$ films, charge separation was proposed to proceed by splitting of an exciton at the porphyrin: $\mathrm{TiO}_{2}$ interface, yielding a charge transfer to the metal oxide. ${ }^{13}$ When the thickness of the porphyrin films becomes larger than a monolayer, the exciton transport to the interface becomes the rate-limiting step for charge generation in such systems. Based on this knowledge, device efficiency could be enhanced by improving exciton transport; for example by better ordering of the porphyrins, ${ }^{12,14,15}$ or by utilizing the longerliving triplet exciton (rather than the singlet exciton) to transfer energy to the interface. ${ }^{16,17}$

In order to further develop the utility of porphyrin-based materials, several groups began to study their introduction into crystalline coordination networks - both metal-organic frameworks (MOFs) ${ }^{18,19}$ and covalent organic frameworks (COFs). ${ }^{20}$ Within the well-defined crystal structures provided by such networks, the transport properties for excitons and charges can be engineered by controlling the chromophore positions 
and orientations within the unit cell. ${ }^{21}$ Furthermore, the mobility of hole versus electron transport could be controlled in a COF by changing the metalation of the porphyrin molecule. ${ }^{20}$

Recently, porphyrin molecules have also been used in surface-anchored metal-organic framework (SURMOF) structures that exhibited photovoltaic action, either with a liquid ${ }^{22,23}$ or solid-state electrolyte. ${ }^{24}$ Further results demonstrated that porphyrin SURMOFs can be effective sensitizers in solid-state triplet-triplet annihilation-based photon upconversion (TTA-UC) systems. ${ }^{25,26}$

While these results highlight the potential of porphyrinbased SURMOFs, the photostability of these molecules is a known concern with respect to their application in other fields, an example being the use as photosensitizers in photodynamic therapy. ${ }^{27-29}$ Chlorins and bacteriochlorins are stable products of light-induced reactions of porphyrins. ${ }^{30-32}$

As understanding the photostability of organic materials is a relevant factor for solar energy harvesting applications, we here examine in detail the changes caused by prolonged illumination of PdP SURMOF thin films. To this end we combine steady-state photoluminescence (PL) and transient absorption spectroscopy (TAS) to study the exciton dynamics of these systems. Additionally, as photoexcitation leads to the generation of triplet excitons, which are paramagnetic and non-thermalised at the point of generation, the dynamics can be traced with transient electron paramagnetic resonance (trEPR) spectroscopy. The combination of results allows us to conclude that prolonged illumination creates sites in the crystalline SURMOF at which triplet excitons are efficiently split, yielding radicals. From these results we can establish a photophysical population model of the radical generation in phototreated PdP SURMOFs.

\section{Results and discussion}

The SURMOF material used in this study is based on the established SURMOF-2 structure ${ }^{22,33}$ and was fabricated using a spraying technique. ${ }^{34}$ In this particular SURMOF, zinc (Zn) paddlewheel nodes are coordinated to 4 ditopic organic linkers (5,15-bis(4carboxyphenyl)-10,20-diphenylporphyrinato) palladium(II) (PdP). Detailed structural information regarding this compound can be found in the literature. ${ }^{25}$ Briefly, the porphyrin linkers are coordinated through the $\mathrm{Zn}$ paddlewheel nodes to form 2D sheets with a square unit cell of $2.5 \mathrm{~nm}$. These sheets stand perpendicular to the substrate and are separated from adjacent sheets by $0.6 \mathrm{~nm}$ (for a schematic drawing see Fig. S12, ESI $\dagger$ ). The close inter-sheet distance results in a good $\pi$-orbital overlap between adjacent porphyrins.

A number of photo-initiated reactions of porphyrins are known, some which proceed even in the absence of oxygen, such as the production of phlorin and chlorin. ${ }^{30-32}$ In the following, we establish how PdP SURMOFs respond to prolonged illumination in oxygen-poor environments (all measurements were performed under dynamic vacuum) and how the photoproducts created by prolonged illumination affect the photophysics of the thin films.
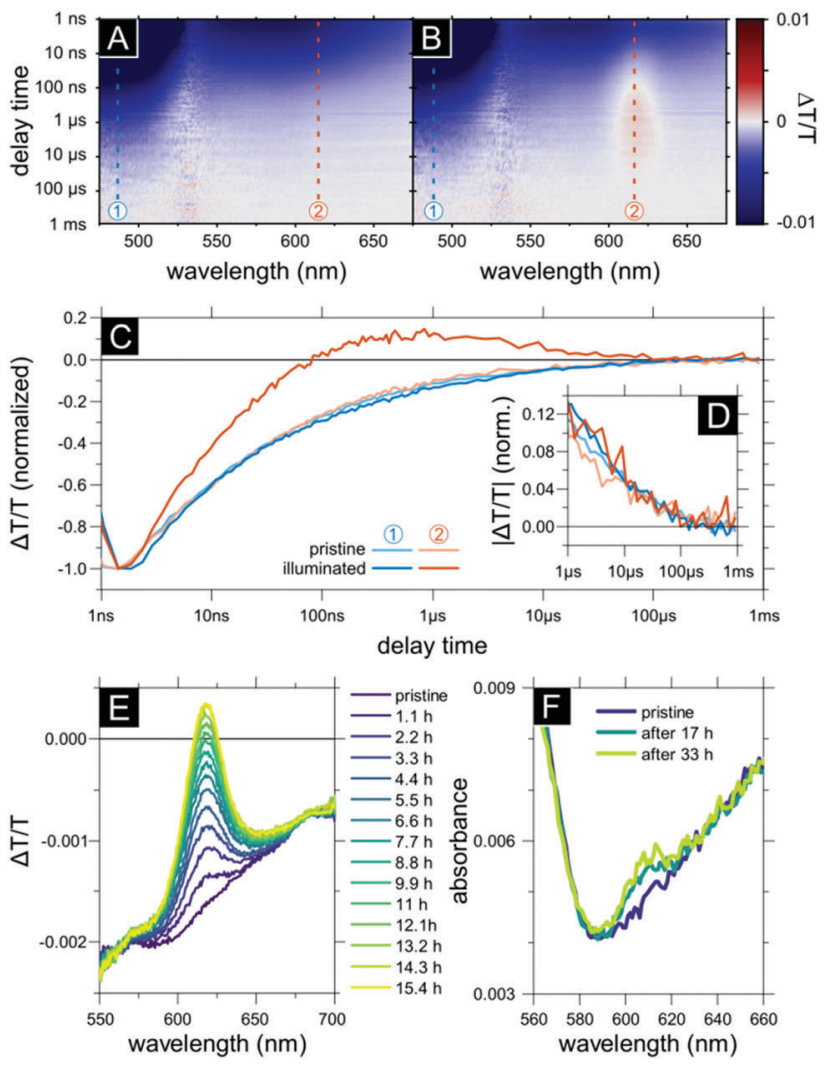

Fig. 1 Effects of prolonged illumination of palladium porphyrin surfaceanchored metal-organic frameworks monitored by transient absorption spectroscopy (TAS) and photoluminescence (PL) spectroscopy. (A) TAS data for a pristine PdP SURMOF and (B) a PdP SURMOF after 15.4 hours of photodegradation with a $532 \mathrm{~nm}$ pulsed pump laser at $120 \mu \mathrm{J} \mathrm{cm} \mathrm{cm}^{-2}$ $\left(500 \mathrm{~Hz}\right.$ repetition rate) under dynamic vacuum $\left(<10^{-4} \mathrm{~Pa}\right)$ at room temperature. (C) Normalized TAS kinetic traces integrated over the tripletdominated region 450-500 $\mathrm{nm}$ (1) and the photoproduct-dominated region 605-620 $\mathrm{nm}$ (2) for the pristine and illuminated samples from panels (A) and (B). (D) Comparison of the absolute values of the kinetic traces in (C) for delay times $>1 \mu \mathrm{s}$. (E) TAS spectra integrated over the time region between $100 \mathrm{~ns}$ and $300 \mathrm{~ns}$. The sample was kept under dynamic vacuum $\left(<10^{-4} \mathrm{~Pa}\right)$ at room temperature and continuously excited with the pulsed $532 \mathrm{~nm}$ pump laser at $120 \mu \mathrm{J} \mathrm{cm}^{-2}$ ( $500 \mathrm{~Hz}$ repetition rate). The shown TAS spectra were taken at a 66 minutes interval. (F) Absorbance spectra of a PdP SURMOF thin film before and after several hours of continuous illumination with a $405 \mathrm{~nm} \mathrm{CW}$ source at $0.1 \mathrm{~W} \mathrm{~cm} \mathrm{~cm}^{-2}$ under dynamic vacuum $\left(<10^{-4} \mathrm{~Pa}\right)$ at room temperature.

In Fig. 1 we compare the photophysics, examined via TAS and steady-state PL, of both pristine films and films that have been exposed to prolonged illumination. In the pristine sample only a single excited-state population can be observed. The spectra at different times (see Fig. S10, ESI $\dagger$ ) do not evolve and can be described by the decay of a single spectrum, thus only a single long-lived excited-state species is created after photoexcitation of the sample. As the Pd-porphyrin is known for its high intersystem crossing yield close to unity ${ }^{35}$ we identify this excited-state species with triplet excitons on the porphyrin molecules. Singlet excitons cannot be observed here due to their lifetime being on the picosecond timescale. ${ }^{22}$ After prolonged illumination, the photophysics significantly changes and reveals 
that two excited-state populations become present. We will now discuss the changes in TAS and steady-state PL results caused by prolonged illumination.

The TAS surfaces of a pristine sample and the same sample after it has been exposed to 15.4 hours of illumination from the $532 \mathrm{~nm}$ pulsed laser source $\left(120 \mu \mathrm{J} \mathrm{cm}^{-2}\right.$ pulse energy, $800 \mathrm{ps}$ pulse length, and $500 \mathrm{~Hz}$ repetition rate) are shown in Fig. 1A and $\mathrm{B}$, respectively. Although the sample was held under dynamic vacuum $\left(<10^{-4} \mathrm{mbar}\right)$, the TAS surfaces before and after the prolonged illumination are significantly different. The clearest difference between the pristine and post-illumination TAS results is the appearance of a positive (absorption bleach) signal in the $605-620 \mathrm{~nm}$ region that appears as the sample is illuminated for prolonged periods. Fig. 1C compares the integrated TAS signal in two wavelength regions - the bleach-region from $605-620 \mathrm{~nm}$ and a region unaffected by illumination from 450-500 $\mathrm{nm}$ - as a function of time for the pristine and illuminated samples. In the pristine sample, there is a photoinduced absorption (PIA) in both regions that decays to zero with the same time constant. This is expected, as all dynamics in this pristine sample track the triplet exciton population; it is the only excited-state population created. After illumination clearly more than one excited-state is created, as the initially observed photoinduced absorption (negative TAS signal) crosses zero into a positive absorption (ground-state bleach, GSB) after roughly $100 \mathrm{~ns}$. Such a zero crossing can be explained only by the existence of more than one excited state population. The absolute values of the normalized TAS kinetics for times $>1 \mu$ s are given in Fig. 1D and highlight that the signals decay identically in both regions. This will be discussed in more detail below, but first we want to establish the nature of the emerging population.

The spectrum of the absorption bleach gives a clue as to the nature of the degradation product created by extended illumination. We observe that the maximum bleach in the 605-620 nm region occurs between 100 and $300 \mathrm{~ns}$ after excitation (Fig. 1B). Therefore, to monitor the creation of the photoproduct, we plot the TAS spectra (integrated in a delay time from 100-300 ns) at increasing illumination times (Fig. 1E). As the illumination time increases, there is a clear increase in the GSB in the 605-620 $\mathrm{nm}$ region. This indicates that the product of the photoreaction caused by prolonged illumination has a ground state absorption band in the region of 605-620 nm, which we can confirm by observing the illumination-induced changes in the absorption spectrum (Fig. 1F, for the full spectrum see Fig. S14 in the ESI $\dagger$ ).

This, along with further evidence from PL-spectroscopy shown below, suggests chlorin as a probable candidate for the product of the photoreaction. The absorption of Pd-chlorin features a maximum around $612 \mathrm{~nm}$, which is in good agreement with the GSB observed in TAS. ${ }^{30}$ The Pd-porphyrin can undergo a photolytic reaction that leads to the reduction of one double bond in the porphyrin macrocycle and thus creates a stable product Pd-chlorin. ${ }^{30}$ This reaction requires a proton source and we suggest that residual solvent (ethanol) or water could play this role. It is known that water molecules form
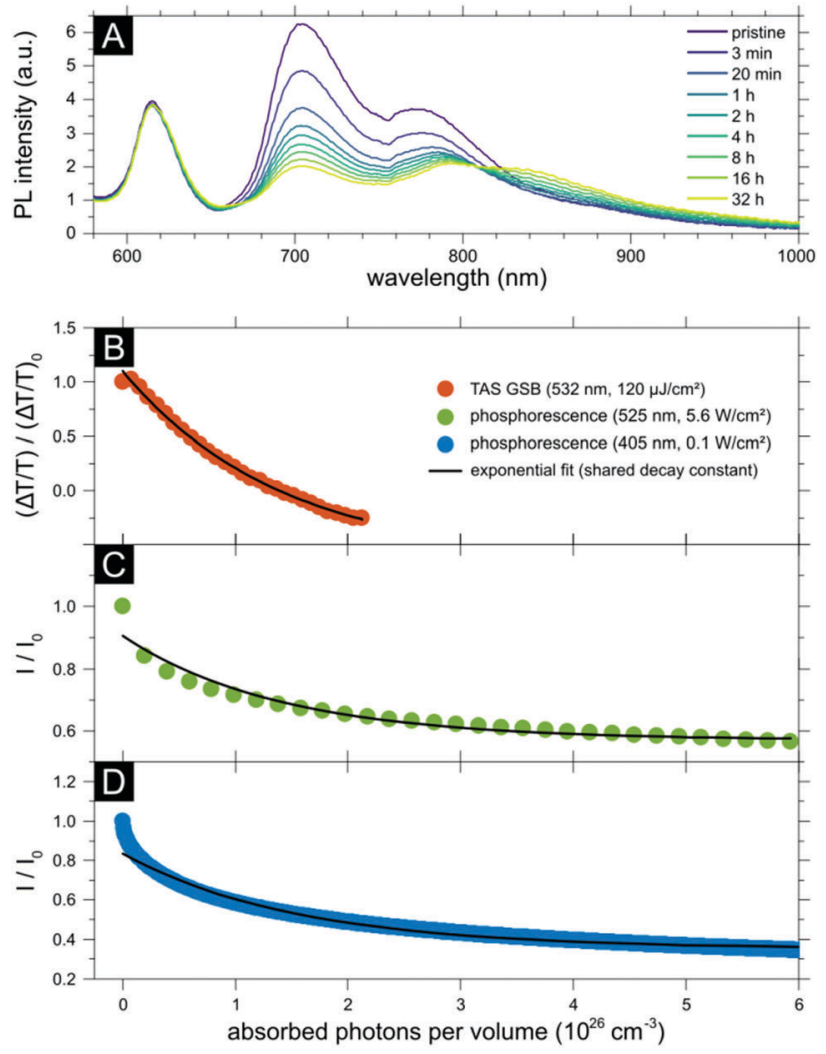

Fig. 2 Changes in PdP SURMOF photoresponse during illumination. (A) PL spectra measured at indicated times during continuous illumination with a $525 \mathrm{~nm} \mathrm{CW}$ laser at $5.6 \mathrm{~W} \mathrm{~cm}^{-2}$ under dynamic vacuum $\left(<10^{-4} \mathrm{~Pa}\right)$ at room temperature. (B) Relative change in the TAS signal in the GSB region (integrated from 605-620 nm). (C) Relative change in phosphorescence (integrated from $700-720 \mathrm{~nm}$ ) during illumination with a $525 \mathrm{~nm}$ CW laser at $5.6 \mathrm{~W} \mathrm{~cm}^{-2}$ at room temperature under dynamic vacuum $\left(<10^{-4} \mathrm{mbar}\right)$. The horizontal axis range of $6 \times 10^{26} \mathrm{~cm}^{-3}$ corresponds to 30 minutes of illumination. (D) Relative change in phosphorescence (integrated from $700-720 \mathrm{~nm}$ ) during illumination with a $405 \mathrm{~nm} \mathrm{CW}$ laser at $0.1 \mathrm{~W} \mathrm{~cm} \mathrm{~cm}^{-2}$ at room temperature under dynamic vacuum $\left(<10^{-4} \mathrm{mbar}\right)$. The horizontal axis range of $6 \times 10^{26} \mathrm{~cm}^{-3}$ corresponds to 22 hours of illumination. The black line represents a mono-exponential fit as a guide to the eye, the decay constant being $1.5 \times 10^{26} \mathrm{~cm}^{-3}$ for all three panels.

hydrogen bonds to the axial positions of the paddle-wheel ${ }^{36}$ and are therefore difficult to remove even under the dynamic vacuum conditions in the TAS and PL measurements. Another hypothesis is that traces of residual ethanol can be directly photo-oxidized to acetaldehyde by porphyrin and, in the absence of oxygen, the hydrogen atoms could reduce the porphyrin, yielding chlorin.

Another indicator for the creation of chlorin can be found when we plot the PL spectrum as a function of illumination time for a PdP SURMOF film (see Fig. 2A). In this case, the pristine film was also kept under dynamic vacuum $\left(<10^{-4} \mathrm{mbar}\right)$ and then illuminated with an intense CW laser ( $\left.525 \mathrm{~nm}, 5.6 \mathrm{~W} \mathrm{~cm}^{-2}\right)$. The $610 \mathrm{~nm}$ peak, coming from the small amount of singlet emission in the PdP before intersystem crossing, remains unchanged by the illumination. However, the phosphorescence peaks from the PdP triplet around 700 and $800 \mathrm{~nm}$ are strongly reduced as the sample is illuminated. Furthermore, the emission 
at wavelengths longer than $800 \mathrm{~nm}$ actually increases upon prolonged illumination. We note that the PL spectrum of Pd-chlorin has - relative to the Pd-porphyrin - a reduced intensity in the porphyrin phosphorescence region whereas it has an increased intensity above $850 \mathrm{~nm} .{ }^{37}$ Thus, the observations of the in situ photoreaction of PdP using TAS and PL spectroscopy are consistent, and both point to the formation of Pd-chlorin as the photoproduct. Nonetheless, for generality we will refer to the molecules created by extended illumination as the "photoproduct"' in the rest of the manuscript.

Before investigating in detail the influence of the photoproduct "dopants" within the PdP SURMOF on its photophysics, we first examine the rate at which the photoproducts are created. In Fig. 2B-D we present the relative change in the TAS bleach signal between 605 and $620 \mathrm{~nm}$ and the relative change in the PdP phosphorescence as a function of the total number of absorbed photons in a given sample volume, $n$. The phosphorescence measurement was performed at a high excitation power density (5.6 $\mathrm{W} \mathrm{cm}^{-2}$, $525 \mathrm{~nm} \mathrm{CW}$; see Fig. 2C) and a much lower excitation power density ( $0.1 \mathrm{~W} \mathrm{~cm}^{-2}, 405 \mathrm{~nm} \mathrm{CW}$, see Fig. 2D). The increase of the photoproduct absorption bleach in the TAS, and the decrease of the PdP PL in both excitation power density regimes follow a very similar trend with respect to the total absorbed photon number per volume (despite the fact that the excitation sources differ in CW/pulsed power density). These data allow us to conclude that the degradation process is independent of the photon energy or power density and instead the number of absorbed photons is the critical factor determining the extent of the photoreaction, which likely occurs when the PdP is in the long-lived triplet excited-state. ${ }^{38}$ The observed change for the TAS and the PL decrease until $6 \times 10^{26} \mathrm{~cm}^{-3}$ absorbed photons (corresponding to 30 minutes in the high and 22 hours in the low power density regime) can be described empirically as proportional to $\exp (-n / \nu)$ with $\nu=(1.53 \pm 0.01) \times$ $10^{26} \mathrm{~cm}^{-3}$. Here $\nu$ is the photon dose required for the fast component of the photoreaction to reach $1 / e$ of its final progress. To put this in perspective, this corresponds to around 50 hours under solar irradiation (under AM1.5 conditions and considering the visible absorption spectrum of a $0.2 \mu \mathrm{m}$ thick PdP SURMOF). The PL continues to degrade after 30 minutes under $5.6 \mathrm{~W} \mathrm{~cm}^{-2}$ excitation, but at a much slower rate (reducing by a further factor of 2 after 32 hours of illumination, as can be seen in Fig. 2A).

Having established that a photoproduct is created, and the absorbed photon density necessary for its significant creation, we now return to studying the influence that the photoproduct has on the photophysics of the sample. We recall that the data presented in Fig. 1 established that the photoproduct leads to a second excited-state. In the following section we use trEPR measurements, to establish the nature of this second photoexcited state created in the illuminated PdP SURMOF. The advantage of trEPR is that it is, different from conventional EPR, very sensitive to short-lived paramagnetic states as they are predicted from the above studies. Such transient states are often associated with emissive and absorptive features in the EPR spectrum reflecting non-equilibrium spin populations. ${ }^{39}$
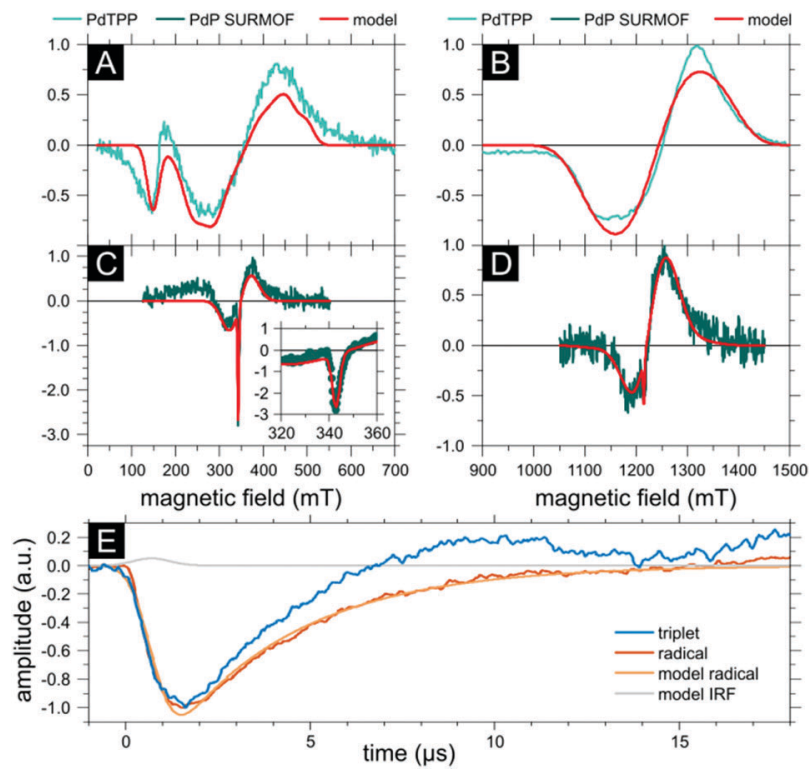

Fig. 3 PdTPP EPR spectra in the X-band (A) and Q-band (B), PdP SURMOF EPR spectra in the $X$-band (C) and $Q$-band (D). The samples were measured at $20 \mathrm{~K}$ with $532 \mathrm{~nm}$ photoexcitation. Simulated model spectra are shown in red. Measured spectra were derived by integrating the first 500 ns of data after the laser flash, then normalizing to unity. Simulations were performed as described in the text, with the fitting parameters given in Table 1. (E) X-band measurement of photoreacted PdP SURMOF, normalized transients for the radical pair and the emissive portion of the triplet signal, with the radical pair decay modelled as a first-order exponential convolved with a Gaussian instrument response function, shown in grey. Transients crossing the zero-line suggest driving by the microwave field and suggest that the true lifetimes are longer than those indicated here.

We investigate the EPR spectra of a heavily photoreacted thin film PdP SURMOF (accumulated exposure on the order of $1 \times 10^{27}$ photons per $\left.\mathrm{cm}^{3}\right)$ in the $\mathrm{X}$ band $(9.6 \mathrm{GHz})$ and $\mathrm{Q}$ band (34 GHz). Long measurement times under constant illumination prevented us from investigating pristine PdP SURMOFs with this method. However, we used a solution of $(5,10,5,20$ tetraphenylporphyrinato)palladium(II) (PdTPP) in toluene as a reference.

Selected EPR spectra along with model fits derived from modelled spin systems ${ }^{40}$ are shown in Fig. 3A-B (PdTPP) and Fig. 3C and D (PdP SURMOF). The simulation parameters are given in Table 1 (full spectra of each measurement and fits to the thermalized spin states are available in the ESI $\dagger$ ). The reference PdTPP solution spectrum has an eee/aaa $(\mathrm{e}=$ emissive, $\mathrm{a}=$ absorptive) pattern, consistent with a triplet excited state populated by intersystem crossing from a photoexcited singlet state.

Table 1 Spin system parameters for the PdTPP triplet as well as the PdP SURMOF triplet and radical pair spin systems. An isotropic $g$-value was chosen for simplicity

\begin{tabular}{llllll}
\hline & $D$ & $E$ & Linewidth & & \\
& $(\mathrm{MHz})$ & $(\mathrm{MHz})$ & $(\mathrm{mT})$ & $g$ & $g_{\text {Strain }}$ \\
\hline PdTPP triplet & 4460 & 700 & 28 & 1.957 & 0.11 \\
PdP SURMOF triplet & 910 & 270 & 37 & 1.977 & 0.137 \\
PdP SURMOF radical pair & - & - & 3.6 & 1.999 & -
\end{tabular}


The absorption and emission features represent microwavedriven transitions between different spin sub-levels of the triplet excited state, arranged in a powder pattern due to the random orientation of the molecules with respect to the direction of the external magnetic field. The spectrum also exhibits a pronounced half-field transition in the X-band measurement. Rapid intersystem crossing is induced by high spin-orbit coupling from the palladium atom in the porphyrin center and leads to a high yield of triplet excited states. ${ }^{41,42}$ The substantial spin-orbit coupling leads also to a large dipolar-coupling value $D=4460 \mathrm{MHz}$ of the observed spin system. The model electron $g$-factor of 1.957 is strongly perturbed from the free electron value $\left(g_{\mathrm{e}}=2.002\right)$, also due to the palladium atom.

In Fig. 3, several changes between the reference spectra (Fig. 3A and B) and those of the illuminated PdP SURMOF (Fig. 3C and D) are observed. Firstly, for the illuminated SURMOF, the width of the triplet exciton spectrum decreases by a factor of almost $5(D=$ $914 \mathrm{MHz}$ ), while the $g$-value of 1.977 is shifted closer to $g_{\mathrm{e}}$. These findings suggest that the SURMOF triplet exciton is more spatially extended and less strongly associated with the palladium atom, compared to the triplet on the isolated PdTPP molecule.

Secondly, and the key finding in relation to establishing the nature of the second excited-state in the photoreacted SURMOF, in addition to a triplet spectrum, the PdP SURMOF trEPR measurements show a sharp emissive feature (see Fig. 3C, inset) which can be modelled as a spin-1/2 particle. This feature indicates the presence of photoexcited radical species. This feature is absent from the measurement of monomeric PdTPP, so its generation is linked to the photodegraded SURMOF environment. The emissive signal, indicative of a non-thermalized spin state population, supports the radical generation mechanism by excited state quenching, rather than by the generation of radicals by, for example, photodegradation. Different radical generation mechanisms are conceivable and cannot be distinguished based on the trEPR results. It is possible that spin-correlated radical pairs are formed upon dissociation of the photoexcited triplet excitons, but short lifetimes or fast spin-relaxation times preclude one radical from being observed in the trEPR spectrum. Alternatively, the generation of a spin-polarised radical in conjunction with the decay of the triplet excitons also provides a credible explanation for the emissive signal. The $g$-factor of the modelled radical pair, 1.999 , is close to $g_{\mathrm{e}}$, and differs substantially from that of the underlying SURMOF triplet exciton, consistent with the radical pair occupying a different lattice position than the triplet. In the Q-band measurement, the radical pair signal is reproducibly less intense, relative to the triplet, than in the X-band. The reason for this difference is not clear yet.

The emergence of a radical signal is consistent with our suggestion of the photoproduct being chlorin. The lowest unoccupied molecular orbital (LUMO) energy levels of porphyrin, chlorin, and bacteriochlorin are nearly isoenergetic. ${ }^{43}$ However, the highest occupied molecular orbital (HOMO) energy levels of chlorin and bacteriochlorin are higher than that of porphyrin. ${ }^{43}$ Such an energy level alignment suggests that an excited-state on the porphyrin could undergo charge transfer at a porphyrinchlorin (or porphyrin-bacteriochlorin) interface with the hole transferring from the porphyrin to the chlorin or bacteriochlorin. This would be consistent with our observations, and we note that this is consistent with reports of the use of chlorin as a hole acceptor in porphyrin-containing arrays. ${ }^{44}$

Having established that the second excited-state species is a radical pair, trEPR can also be used to make limited conclusions regarding the temporal evolution of the radical and triplet species in the SURMOF. A high microwave field was used during measurement to obtain a good signal-to-noise ratio, and this can drive the decay of spin polarization, obscuring the true dynamics. Similarly the $20 \mathrm{~K}$ sample temperature, also used to improve signals, is expected to change the measured lifetimes compared to room temperature measurements. However, the time-resolved data are nonetheless broadly consistent with the findings of the TAS and PL studies. The weaker radical signal and faster triplet thermalization in the Q-band measurement make modelling the radical decay difficult, so we focus our attention on the X-band data. Here, as shown in Fig. 3E, the radical and triplet signals rise concurrently, consistent with triplet-to-charge conversion taking place within the trEPR instrument response time of several hundred nanoseconds. Finally, the radical signal decay is wellmodelled as a first-order exponential process with a lifetime of $3.5 \mu \mathrm{s}$, which we will find below to be consistent with the radical population decay observed in TAS.

Having been established by trEPR that the excited state introduced by photoreaction of the PdP SURMOF is a radical state, we proceed to examine the question of how this radical state is formed. To this end we return to TAS and investigate in detail a heavily photoreacted thin film ( $>100$ hours, accumulated exposure on the order of $10^{28}$ photons $\mathrm{cm}^{-3}$ ).

In Fig. 4 we show the TAS kinetic traces as a function of delay time for a photoreacted PdP SURMOF in two wavelength

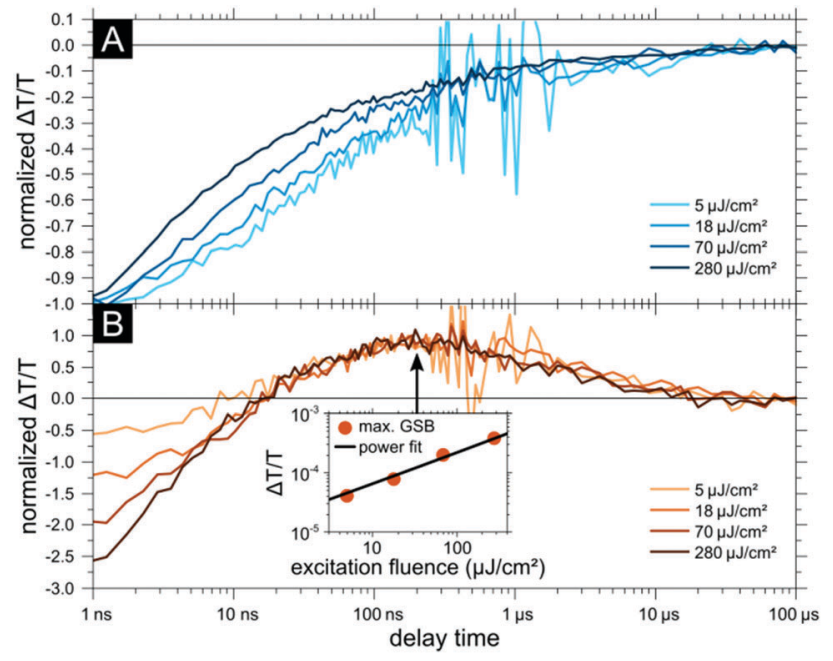

Fig. 4 Excitation fluence dependence of transient absorption measurements under dynamic vacuum $\left(<10^{-4} \mathrm{~Pa}\right)$ at room temperature. (A) Integrated triplet PIA kinetics (450-500 nm) show a strong fluence-dependence. (B) In contrast, the decay of the integrated charge-induced GSB kinetics $(605-620 \mathrm{~nm})$ does not change with varying excitation intensities. The inset shows how the radical GSB maximum signal (at around 200 ns) scales with excitation fluence. The maximum follows a power law with an exponent of 0.5 (black line). 
ranges for several excitation fluences. The first wavelength range is where the signal is dominated by PIA of the PdP triplet exciton state (450-500 nm, Fig. 4A). The second wavelength range is where the signal is influenced both by PIA of the PdP triplet exciton, and also by the GSB associated with a radical state on the photoproduct (605-620 nm, Fig. 4B). As the initial excited-state density in the sample is increased by increasing the excitation fluence, the decay of the triplet population becomes faster. This establishes that even in the photoreacted sample, triplet-triplet annihilation (TTA) changes the rate at which PdP triplet excitons decay to the ground state at early time ( $<100 \mathrm{~ns}$ ). In fact, the PdP triplet recombination kinetics (measured in the $450-500 \mathrm{~nm}$ wavelength range) are almost identical before and after extended illumination (see Fig. 1C). This suggests that only a minor fraction of the PdP triplet population is quenched by charge transfer to the photoproduct, and the major recombination mechanism for PdP triplets remains TTA under the excitation conditions used for TAS.

Fig. $4 \mathrm{~B}$ displays the intensity dependence in the $605-620 \mathrm{~nm}$ region wherein the initial PIA from the PdP triplet population is overwhelmed by a net bleach as the triplet population is replaced by the radical population. As the radical pair state is comprised of a radical on a PdP and photoproduct molecule, we observe a bleach of the photoproduct absorption, described earlier in Fig. 1. The TAS signals for the different fluences are normalized to the bleach maxima (which are reached around $200 \mathrm{~ns}$ after photoexcitation). In the time period before $200 \mathrm{~ns}$, a fluence dependence of the signal can be observed that can be attributed to the higher yield of radical pair states when the TTA annihilation rate is not so high. The yield of radical pair state changes decreases by a factor of 5 as the excitation fluence increases by a factor of 56. In fact, the yield of the radical pair state scales with the square root of the excitation fluence, as can be seen by the dependence of the GSB maximum around $200 \mathrm{~ns}$ on excitation fluence (see the inset of Fig. 3B). This again indicates that the radical pair states are created from the PdP triplet state, but the primary loss channel from the PdP state is TTA and only a small fraction of the PdP excited states form radical pairs.

Further support for the radical pair formation being a minor loss channel comes from a comparison of the decay dynamics of the PdP triplet and the radical pair state populations. In Fig. 1C TAS kinetics are compared for the PdP tripletdominated region (450-500 $\mathrm{nm})$ and the region dominated by the photoproduct-GSB $(605-620 \mathrm{~nm})$, both in a pristine film and a film after prolonged illumination. The pre- and postillumination triplet kinetics are identical, suggesting that only a minor fraction of the PdP triplets form radical pairs.

Furthermore, after around $5 \mu \mathrm{s}$, the radical pair signal decay exactly matches the decay of the PdP triplets (Fig. 1D). All four kinetic traces in Fig. 1D can be fit with a monoexponential decay function with a lifetime of $(3.7 \pm 0.3) \mu$ s, which is in agreement with the radical population lifetime found by trEPR. However, this merely describes the effective lifetime of the triplet population from which the radical pairs are populated and should not be confused with the lifetime of a single radical pair itself.
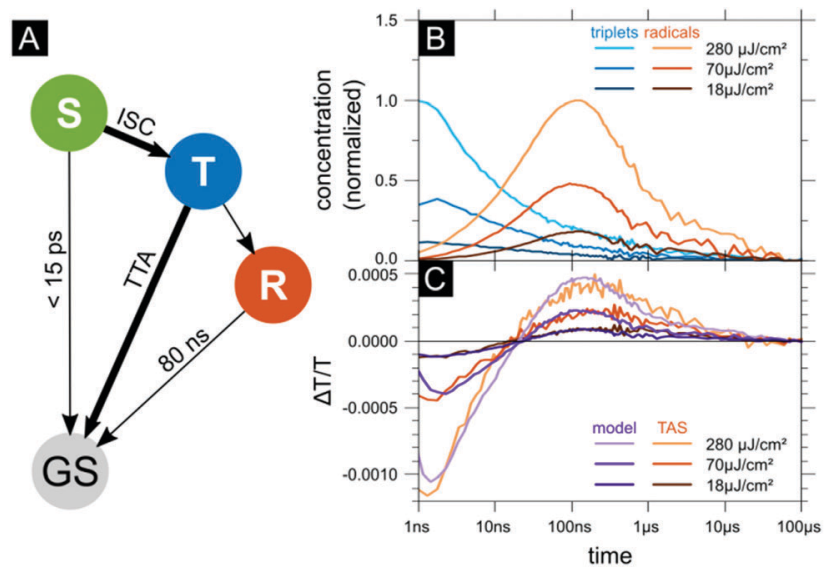

Fig. 5 Model of PdP SURMOF photoresponse. (A) Schematic model showing how excited states can move between singlet (S) and triplet (T) exciton states, radical pair states (R) and the ground state (GS). (B) Model of the radical pair population dynamics. The triplet population is taken directly from TAS and not modelled. The populations are normalized to the maximum of the highest excitation fluence kinetic $\left(280 \mu \mathrm{J} \mathrm{cm}{ }^{-2}\right)$. (C) Comparison of modelled and experimental TAS kinetics in the GSB-dominated wavelength region $605-620 \mathrm{~nm}$.

The agreement of both population decays suggests that the radical lifetime is short compared to that of the PdP triplet.

The qualitative considerations made so far allow us to build a quantitative model to fit the observed TAS data. A graphical representation is shown in Fig. 5A. After photo-induced formation of a singlet exciton state (S), these excitons can either decay back to the ground state (GS) or perform intersystem crossing (ISC) into the triplet state (T). The mobile triplet population is emptied predominantly by TTA (monomolecular decay can be neglected altogether at these excitation fluences). However, a fraction of the diffusing triplet excitons will encounter an illumination-induced photoproduct site and form a radical pair state (R), which quickly recombines to the ground state. The rate equation describing the radical pair dynamics is given by

$$
\frac{\mathrm{d} R}{\mathrm{~d} t}=+k_{\mathrm{T} \rightarrow \mathrm{R}} T(t)-k_{\mathrm{R} \rightarrow \mathrm{GS}} R(t),
$$

where $T$ and $R$ are the triplet and radical pair concentrations, respectively, $k_{\mathrm{T} \rightarrow \mathrm{R}}$ is the rate at which triplets generate radical pairs and $k_{\mathrm{R} \rightarrow \mathrm{GS}}$ is the inverse lifetime of the radical pairs. The equation can be solved analytically:

$$
R(t)=k_{\mathrm{T} \rightarrow \mathrm{R}} \mathrm{e}^{-k_{\mathrm{R} \rightarrow \mathrm{GS}} t} \int_{0}^{t} \mathrm{e}^{-k_{\mathrm{R} \rightarrow \mathrm{GS}} \xi} T(\xi) \mathrm{d} \xi .
$$

The triplet concentration is proportional to the TAS signal. The triplet-to-radical-pair rate $k_{\mathrm{T} \rightarrow \mathrm{R}}$ acts as a scaling factor in the equation, as the relative cross-sections of the PdP triplets and radical pairs are unknown, the experimental data do not constrain $k_{\mathrm{T} \rightarrow \mathrm{R}}$ and we cannot obtain absolute values for the radical pair concentration. However, using eqn (2) to fit the data does allow $k_{\mathrm{R} \rightarrow \mathrm{GS}}$ to be precisely extracted and we thereby gain insights into the relative population dynamics.

We calculate the development of the relative radical pair population for three excitation fluences (Fig. 5B) and obtain 
from this the single-species spectra (see Fig. S9 in the ESI $\dagger$ ) and a model of the TAS kinetics. A rate of $k_{\mathrm{R} \rightarrow \mathrm{GS}}=12.5 \times 10^{6} \mathrm{~s}^{-1}$ (a lifetime of $80 \mathrm{~ns}$ ) results in the best match between model and experimental data (shown exemplarily for the GSB-dominated wavelength region 605-620 nm in Fig. 5C). Furthermore, we see in Fig. 5B that the relative yield of radical pairs (the maximum radical pair concentration divided by the maximum triplet concentration) increases as the excitation fluence is reduced. This is consistent with the 0.5 power-law scaling qualitatively observed, and caused by the fact that radical pair generation competes with TTA that is less dominant at lower excitation power densities.

\section{Summary}

We have investigated the photophysical effects of prolonged illumination on a surface-anchored metal-organic framework based on a palladium porphyrin linker. Based on monitoring the photoreaction in situ with TAS and steady-state PL measurements we present evidence that a photoproduct is formed, which is likely the respective Pd-chlorin. Comparing the creation of the photoproduct bleach in the TAS and the disappearance of the PdP phosphorescence we establish a rate for the creation of the photoproduct of $1.4 \times 10^{27}$ photons per $\mathrm{cm}^{3}$.

Using trEPR we establish that the additional excited-state created in the photoreacted sample is a radical, and returning to TAS we find that radicals are created when the PdP triplet reaches a photoproduct molecule and an electron transfer takes place between the two molecules.

We find that the concentration of photoproduct sites in the SURMOF is insufficient to change the triplet exciton population dynamics significantly. The radicals are short-lived (80 ns lifetime) and their population closely follows the precursor triplet population.

Applications of PdP SURMOFs will benefit from our findings. On one hand, the photogeneration of radical pairs represents a loss channel for applications relying on large triplet exciton concentrations (such as TTA-UC) and needs to be addressed in the design process. On the other hand, the radicals could be a welcome product for photovoltaic application, given that an efficient charge transport pathway can be introduced.

\section{Experimental section}

\section{Materials}

Zinc acetate and ethanol were purchased from VWR and used as received. The PdP linker was synthesized starting from 5,15-bis((4'-methoxycarbonyl)phenyl)-10,20-diphenylporphyrin as described in the ESI. $\dagger^{45}$

\section{Sample preparation}

The PdP SURMOFs were fabricated by spray coating using a zinc acetate solution in ethanol $\left(1 \mathrm{mM} \mathrm{L}^{-1}\right)$ and a PdP linker solution in ethanol $\left(20 \mu \mathrm{M} \mathrm{L}^{-1}\right)$. The zinc acetate solution was sprayed on the substrate (for $15 \mathrm{~s}$ ), followed by a waiting time (35 s) and rinsing with pure ethanol (5 s). Then the linker solution was sprayed on top (25 s) followed by a waiting time (35 s) and rinsing with pure ethanol $(5 \mathrm{~s})$. These steps were repeated 15 or 30 times. The growth rate was previously determined to be around $16 \mathrm{~nm}$ per cycle. ${ }^{25}$ The crystallinity of the thin film was ascertained by X-ray diffraction (XRD) characterization (Bruker D8 Advance).

\section{Absorption spectroscopy}

Absorption spectra were measured with a Perkin-Elmer Lambda $950 \mathrm{UV} / \mathrm{vis} / \mathrm{NIR}$ spectrophotometer in the absorbance mode. The PdP linker solution was measured in the transmission mode with the cuvette placed in the beam path. The thin-film PdP SURMOF sample was measured inside an integrating sphere.

\section{Transient absorption spectroscopy (TAS)}

Nanosecond transient absorption spectroscopy (TAS) was performed with a custom-built pump-probe system. The output of a titanium:sapphire (Ti:Sa) femtosecond amplifier (SpectraPhysics Spitfire Pro XP, center wavelength $800 \mathrm{~nm}$, repetition rate $1 \mathrm{kHz}$ ) was used to generate a white light continuum (probe beam) inside a sapphire crystal. The pump beam was generated from an oscillator/amplifier sub-nanosecond laser system (Innolas Picolo AOT 1 MOPA, center wavelength $532 \mathrm{~nm}$, repetition rate $500 \mathrm{~Hz}$, pulse width $800 \mathrm{ps}$ ). The pump laser was electronically triggered by the Ti:Sa amplifier, the pump-probe delay could be set by delaying this trigger signal using a digital delay generator (Stanford Research Systems DG535). To prevent triplet quenching by atmospheric oxygen, the sample was kept under dynamic vacuum with pressure less than $10^{-4} \mathrm{~Pa}$. After passing the sample, the probe beam was dispersed on a flint glass prism and detected at $1 \mathrm{kHz}$ on a linear image sensor (Hamamatsu NMOS S3904-512Q). Each probe pulse from the laser was read out into computer software and adjacent readings were used to calculate the transient absorption data, $\Delta T / T$ (differential transmission).

\section{Time-resolved photoluminescence spectroscopy}

Time resolved photoluminescence (PL) spectroscopy was performed with the same excitation laser (532 nm) as used in the TAS measurements. The PL signal was detected with a streak camera (Hamamatsu Universal Streak Camera C10910-02 with slow single sweep unit M10913 and Acton SpectraPro SP2300 spectrometer). The sample was again kept under dynamic vacuum with pressures less than $10^{-4} \mathrm{~Pa}$.

\section{Steady-state PL spectroscopy}

The sample was excited with a $525 \mathrm{~nm}$ continuous wave (CW) laser diode (Roithner LaserTechnik LD-515-10MG, tuned to $525 \mathrm{~nm}$ ) and the emission spectra were measured with a fibercoupled CCD spectrometer (Avantes AvaSpec ULS-RS-TEC). The sample was again kept under dynamic vacuum with pressures less than $10^{-4} \mathrm{~Pa}$.

\section{Time-resolved EPR spectroscopy}

A laboratory-built time-resolved electron paramagnetic resonance (trEPR) spectrometer was used to study photogenerated paramagnetic states generated in the PdP SURMOF, and in 
toluene solution containing $0.5 \mathrm{mM}$ PdTPP. Measurements were carried out with X-band $(9.6 \mathrm{GHz})$ and Q-band $(34 \mathrm{GHz})$ microwave radiation. Deaerated samples were sealed in quartz capillary tubes under a helium atmosphere and placed in a microwave resonator (Bruker ER 4118X-MD5 for the X-band or a home-built Q-band cavity resonator, both equipped with an optical access window), housed inside a helium gas-flow cryostat cooled to $20 \mathrm{~K}$. This was situated within the poles of an electromagnet. Optical excitation at $\lambda=532 \mathrm{~nm}$ was provided by a diode-pumped Nd:YAG laser (Atum Laser Titan AC compact $15 \mathrm{MM})$ equipped with a second harmonic generator $(100 \mathrm{~Hz}$ repetition rate, $2 \mathrm{~mJ}$ pulse energy, spot size approximately $1 \mathrm{~cm}^{2}$, pulse length $5 \mathrm{~ns}$ ). Time-traces showing microwave absorption and emission after laser excitation, spanning $20 \mu \mathrm{s}$ (X-band) and $10 \mu \mathrm{s}$ (Q-band), were recorded using a digital oscilloscope (LeCroy WaveRunner 104MXi) as a function of external magnetic field strength. Several EPR transients were accumulated for each fieldposition. The non-resonant background signal induced by the laser flash was recorded far from the resonant signal and subtracted from each EPR transient. The magnetic field axis was calibrated using a NMR Gaussmeter (Bruker ER 035M). The (constant) offset between the positions of the Gaussmeter and the sample was determined by a reference sample (nitrogen encapsulated in C60). One-dimensional spectra were produced by integrating the first $500 \mathrm{~ns}$ of the signal immediately following the laser flash. These were simulated using the powder pattern module "pepper" from the simulation toolbox Easyspin ${ }^{46}$ for Matlab. The spin systems were fitted to the X- and Q-band spectra simultaneously.

\section{MALDI-ToF}

MALDI-ToF/ToF-MS experiments were carried out using a 4800 Plus MALDI-ToF/ToF mass spectrometer (Applied Biosystems/ MDS SCIEX, Foster City, CA, USA) equipped with a Nd:YAG pulsed laser (355 nm wavelength of $<500 \mathrm{ps}$ pulse and $200 \mathrm{~Hz}$ repetition rate). The 4000 Series Explorer software (V 3.5.3, Build 1017, 2007, Foster City, CA, USA) and the Data Explorer software (V 4.9, Build 115, 2007, Foster City, CA, USA) were used for analysis. Data acquisition was performed in the reflector positive ion mode. Each mass spectrum obtained was an average of 500 laser shots over the whole spot.

\section{$\mathrm{X}$-ray diffraction}

The XRD measurements in out-of-plane geometry (co-planar orientation) were carried out using a Bruker D8-Advance diffractometer equipped with a position sensitive detector Lynxeye in $\theta-\theta$ geometry. A variable divergence slit and $2.3^{\circ}$ Soller-slit were used on the secondary side. A Cu-anode with $\mathrm{Cu} \mathrm{K} \alpha_{1,2^{-}}$ radiation $(\lambda=0.154018 \mathrm{~nm})$ was used as the source.

\section{Author contributions}

M. A., B. S. R., and I. A. H. conceived and designed the experiments with the assistance from C. W. on matters relating to the SURMOF fabrication and K. L. and R. W. M. on matters relating to the trEPR measurements. The PdP linkers were designed and synthesized by G. E. and M. O. S.; N. B. and M. O. prepared the PdP SURMOF samples with the guidance of R. H. and C. W. R. H. performed X-ray diffraction and MALDIToF experiments. M. A. performed absorption spectroscopy, TAS experiments, steady-state PL spectroscopy, and analyzed the acquired data. D. B. and A. T. assisted with the optical measurements and SURMOF chemistry respectively. F. K. and R. W. M. performed and analyzed the trEPR experiments under the guidance of K. L. and J. B. The paper was written by M. A. and I. A. H. and all authors discussed the results and commented on the paper.

\section{Conflicts of interest}

There are no conflicts to declare.

\section{Acknowledgements}

This work was supported by the Helmholtz Program Science and Technology of Nanosystems (STN) and the Helmholtz Energy Materials Foundry (HEMF). M. A. acknowledges support from Karlsruhe School of Optics and Photonics (KSOP) graduate school. I. A. H. and M. A. thank the Baden-Württemberg Stiftung for financial support. M. O. S. acknowledges grant support from Science Foundation Ireland (IvP 13/IA/1894). B. S. R. would like to acknowledge the financial support provided by Helmholtz Recruitment Initiative Fellowship. R. W. M. acknowledges financial support from the Helmholtz Association. We gratefully acknowledge Dr Murad J. Y. Tayebjee and the German Academic Exchange Service (DAAD) for collection of the X-band measurement of PdTPP in toluene solution. F. K. and J. B. gratefully acknowledge financial support from the DFG (SPP 1601). I. A. H., R. H. and C. W. acknowledge funding from German DFG, SPP 1928 COORNETS.

\section{References}

1 R. Croce and $\mathrm{H}$. van Amerongen, Natural strategies for photosynthetic light harvesting, Nat. Chem. Biol., 2014, 10, 492-501.

2 E. Romero, V. I. Novoderezhkin and R. van Grondelle, Quantum design of photosynthesis for bio-inspired solarenergy conversion, Nature, 2017, 543, 355-365.

3 J. S. Lindsey, De Novo Synthesis of Gem-Dialkyl Chlorophyll Analogues for Probing and Emulating Our Green World, Chem. Rev., 2015, 115, 6534-6620.

4 B. A. Gregg, M. A. Fox and A. J. Bard, Photovoltaic effect in symmetrical cells of a liquid crystal porphyrin, J. Phys. Chem., 1990, 94, 1586-1598.

5 P. G. Schouten, J. M. Warman, M. P. de Haas, M. A. Fox and H.-L. Pan, Charge migration in supramolecular stacks of peripherally substituted porphyrins, Nature, 1991, 353, 353736a0.

6 C. Liu, H. Pan, M. A. Fox and A. J. Bard, Reversible Charge Trapping/Detrapping in a Photoconductive Insulator of 
Liquid Crystal Zinc Porphyrin, Chem. Mater., 1997, 9, 1422-1429.

7 A. D. Schwab, D. E. Smith, B. Bond-Watts, D. E. Johnston, J. Hone, A. T. Johnson, J. C. de Paula and W. F. Smith, Photoconductivity of Self-Assembled Porphyrin Nanorods, Nano Lett., 2004, 4, 1261-1265.

8 A. P. Schall, P. Iavicoli, Z. J. Qi, J. Menko, Y. Lu, M. Linares, J. C. de Paula, D. B. Amabilino, A. T. Johnson and W. F. Smith, Photoconductivity of Nanofilaments That are Self-Assembled from a Porphyrin with Long Alkyl-Chain Substituents, J. Phys. Chem. C, 2015, 119, 26154-26163.

9 A. A. Ryan and M. O. Senge, How green is green chemistry? Chlorophylls as a bioresource from biorefineries and their commercial potential in medicine and photovoltaics, Photochem. Photobiol. Sci., 2015, 14, 638-660.

10 T. Higashino and H. Imahori, Porphyrins as excellent dyes for dye-sensitized solar cells: recent developments and insights, Dalton Trans., 2015, 44, 448-463.

11 A. Huijser, T. J. Savenije, A. Kotlewski, S. J. Picken and L. D. A. Siebbeles, Efficient Light-Harvesting Layers of Homeotropically Aligned Porphyrin Derivatives, Adv. Mater., 2006, 18, 2234-2239.

12 A. Huijser, T. J. Savenije, S. C. J. Meskers, M. J. W. Vermeulen and L. D. A. Siebbeles, The Mechanism of Long-Range Exciton Diffusion in a Nematically Organized Porphyrin Layer, J. Am. Chem. Soc., 2008, 130, 12496-12500.

13 J. E. Kroeze, T. J. Savenije and J. M. Warman, Electrodeless Determination of the Trap Density, Decay Kinetics, and Charge Separation Efficiency of Dye-Sensitized Nanocrystalline $\mathrm{TiO}_{2}$, J. Am. Chem. Soc., 2004, 126, 7608-7618.

14 A. Huijser, B. M. J. M. Suijkerbuijk, R. J. M. Klein Gebbink, T. J. Savenije and L. D. A. Siebbeles, Efficient Exciton Transport in Layers of Self-Assembled Porphyrin Derivatives, J. Am. Chem. Soc., 2008, 130, 2485-2492.

15 L. D. A. Siebbeles, A. Huijser and T. J. Savenije, Effects of molecular organization on exciton diffusion in thin films of bioinspired light-harvesting molecules, J. Mater. Chem., 2009, 19, 6067.

16 J. E. Kroeze, R. B. M. Koehorst and T. J. Savenije, Singlet and Triplet Exciton Diffusion in a Self-Organizing Porphyrin Antenna Layer, Adv. Funct. Mater., 2004, 14, 992-998.

17 J. E. Kroeze, T. J. Savenije and J. M. Warman, Efficient Charge Separation in a Smooth- $\mathrm{TiO}_{2} /$ Palladium-Porphyrin Bilayer via Long-Distance Triplet-State Diffusion, Adv. Mater., 2002, 14, 1760-1763.

18 H.-J. Son, S. Jin, S. Patwardhan, S. J. Wezenberg, N. C. Jeong, M. So, C. E. Wilmer, A. A. Sarjeant, G. C. Schatz, R. Q. Snurr, O. K. Farha, G. P. Wiederrecht and J. T. Hupp, LightHarvesting and Ultrafast Energy Migration in PorphyrinBased Metal-Organic Frameworks, J. Am. Chem. Soc., 2013, 135, 862-869.

19 C. Y. Lee, O. K. Farha, B. J. Hong, A. A. Sarjeant, S. T. Nguyen and J. T. Hupp, Light-Harvesting Metal-Organic Frameworks (MOFs): Efficient Strut-to-Strut Energy Transfer in Bodipy and Porphyrin-Based MOFs, J. Am. Chem. Soc., 2011, 133, 15858-15861.
20 X. Feng, L. Liu, Y. Honsho, A. Saeki, S. Seki, S. Irle, Y. Dong, A. Nagai and D. Jiang, High-Rate Charge-Carrier Transport in Porphyrin Covalent Organic Frameworks: Switching from Hole to Electron to Ambipolar Conduction, Angew. Chem., 2012, 124, 2672-2676.

21 P. Deria, J. Yu, R. P. Balaraman, J. Mashni and S. N. White, Topology-dependent emissive properties of zirconium-based porphyrin MOFs, Chem. Commun., 2016, 52, 13031-13034.

22 J. Liu, W. Zhou, J. Liu, I. Howard, G. Kilibarda, S. Schlabach, D. Coupry, M. Addicoat, S. Yoneda, Y. Tsutsui, T. Sakurai, S. Seki, Z. Wang, P. Lindemann, E. Redel, T. Heine and C. Wöll, Photoinduced Charge-Carrier Generation in Epitaxial MOF Thin Films: High Efficiency as a Result of an Indirect Electronic Band Gap?, Angew. Chem., Int. Ed., 2015, 54, 7441-7445.

23 W. A. Maza, A. J. Haring, S. R. Ahrenholtz, C. C. Epley, S. Y. Lin and A. J. Morris, Ruthenium(II)-polypyridyl zirconium(Iv) metal-organic frameworks as a new class of sensitized solar cells, Chem. Sci., 2016, 7, 719-727.

24 J. Liu, W. Zhou, J. Liu, Y. Fujimori, T. Higashino, H. Imahori, X. Jiang, J. Zhao, T. Sakurai, Y. Hattori, W. Matsuda, S. Seki, S. K. Garlapati, S. Dasgupta, E. Redel, L. Sun and C. Wöll, A new class of epitaxial porphyrin metalorganic framework thin films with extremely high photocarrier generation efficiency: promising materials for all-solid-state solar cells, J. Mater. Chem. A, 2016, 4, 12739-12747.

25 M. Oldenburg, A. Turshatov, D. Busko, S. Wollgarten, M. Adams, N. Baroni, A. Welle, E. Redel, C. Wöll, B. S. Richards and I. A. Howard, Photon Upconversion at Crystalline Organic-Organic Heterojunctions, Adv. Mater., 2016, 28, 8477-8482.

26 J. Park, M. Xu, F. Li and H.-C. Zhou, 3D Long-Range Triplet Migration in a Water-Stable Metal-Organic Framework for Upconversion-Based Ultralow-Power in Vivo Imaging, J. Am. Chem. Soc., 2018, 140, 5493-5499.

27 T. S. Mang, T. J. Dougherty, W. R. Potter, D. G. Boyle, S. Somer and J. Moan, Photobleaching of porphyrins used in photodynamic therapy and implications for therapy, Photochem. Photobiol., 1987, 45, 501-506.

28 S. Luo, E. Zhang, Y. Su, T. Cheng and C. Shi, A review of NIR dyes in cancer targeting and imaging, Biomaterials, 2011, 32, 7127-7138.

29 L. G. Arnaut, in Advances in Inorganic Chemistry, ed. R. van Eldik and G. Stochel, Academic Press, 2011, vol. 63, pp. 187-233.

30 K. Kalyanasundaram, Mechanism of photoreduction of water-soluble palladium and zinc porphyrins, J. Photochem. Photobiol., A, 1988, 42, 87-109.

31 N. N. Sergeeva and M. O. Senge, in CRC Handbook of Organic Photochemistry and Photobiology, ed. A. G. Griesbeck, M. Oelgemöller and F. Ghetti, CRC Press, Boca Raton, FL, USA, 2012, pp. 831-876.

32 D. Mauzerall, The Photoreduction of Porphyrins: Structure of the Products, J. Am. Chem. Soc., 1962, 84, 2437-2445.

33 J. Liu, B. Lukose, O. Shekhah, H. K. Arslan, P. Weidler, H. Gliemann, S. Bräse, S. Grosjean, A. Godt, X. Feng, K. Müllen, I.-B. Magdau, T. Heine and C. Wöll, A novel series 
of isoreticular metal organic frameworks: realizing metastable structures by liquid phase epitaxy, Sci. Rep., 2012, 2, 921.

34 H. K. Arslan, O. Shekhah, J. Wohlgemuth, M. Franzreb, R. A. Fischer and C. Wöll, High-Throughput Fabrication of Uniform and Homogenous MOF Coatings, Adv. Funct. Mater., 2011, 21, 4228-4231.

35 K. N. Solov'ev, M. P. Tsvirko and V. V. Sapunov, Quantum yield for intersystem crossing in nonfluorescent metal porphyrins, J. Appl. Spectrosc., 1973, 18, 543-545.

36 H. K. Arslan, O. Shekhah, D. C. F. Wieland, M. Paulus, C. Sternemann, M. A. Schroer, S. Tiemeyer, M. Tolan, R. A. Fischer and C. Wöll, Intercalation in Layered MetalOrganic Frameworks: Reversible Inclusion of an Extended $\pi$-System, J. Am. Chem. Soc., 2011, 133, 8158-8161.

37 J. Schneider, K. Q. Vuong, J. A. Calladine, X.-Z. Sun, A. C. Whitwood, M. W. George and R. N. Perutz, Photochemistry and Photophysics of a Pd(II) Metalloporphyrin: Re(I) Tricarbonyl Bipyridine Molecular Dyad and its Activity Toward the Photoreduction of $\mathrm{CO}_{2}$ to CO, Inorg. Chem., 2011, 50, 11877-11889.

38 S. Schmidbauer, A. Hohenleutner and B. König, Chemical Degradation in Organic Light-Emitting Devices: Mechanisms and Implications for the Design of New Materials, $A d v$. Mater., 2013, 25, 2114-2129.

39 C. D. Buckley, D. A. Hunter, P. J. Hore and K. A. McLauchlan, Electron spin resonance of spin-correlated radical pairs, Chem. Phys. Lett., 1987, 135, 307-312.
40 C. A. Hutchison and B. W. Mangum, Paramagnetic Resonance Absorption in Naphthalene in Its Phosphorescent State, J. Chem. Phys., 1958, 29, 952-953.

41 J. A. Kooter and G. W. Canters, Zeeman and optical experiments on the lowest triplet state of palladium porphin, Mol. Phys., 1980, 41, 361-375.

42 H. Miwa, K. Ishii and N. Kobayashi, Electronic Structures of Zinc and Palladium Tetraazaporphyrin Derivatives Controlled by Fused Benzo Rings, Chem. - Eur. J., 2004, 10, 4422-4435.

43 C. K. Chang, L. K. Hanson, P. F. Richardson, R. Young and J. Fajer, Cation radicals of ferrous and free base isobacteriochlorins: Models for siroheme and sirohydrochlorin, Proc. Natl. Acad. Sci. U. S. A., 1981, 78, 2652-2656.

44 G. Hu, H. Suk Kang, A. Kumar Mandal, A. Roy, C. Kirmaier, D. F. Bocian, D. Holten and J. S. Lindsey, Synthesis of arrays containing porphyrin, chlorin, and perylene-imide constituents for panchromatic light-harvesting and charge separation, RSC Adv., 2018, 8, 23854-23874.

45 M. B. Bakar, M. Oelgemöller and M. O. Senge, Lead structures for applications in photodynamic therapy. Part 2: Synthetic studies for photo-triggered release systems of bioconjugate porphyrin photosensitizers, Tetrahedron, 2009, 65, 7064-7078.

46 S. Stoll and A. Schweiger, EasySpin, a comprehensive software package for spectral simulation and analysis in EPR, J. Magn. Reson., 2006, 178, 42-55. 